\title{
NEW OSCILLATION CRITERIA FOR SECOND ORDER NEUTRAL TYPE DIFFERENCE EQUATIONS
}

\author{
R. Kodeeswaran ${ }^{1}$ and R. Nagarajan ${ }^{2}$ \\ 1,2 Department of Mathematics, Kandaswami Kandar's College, \\ Velur - 638 182, Namakkal (Dt), Tamil Nadu, India. \\ 1E-Mail Address: srkodeesh@gmail.com , ²E-Mail Address: kneenagu@gmail.com
}

\section{ABSTRACT}

In this paper, we present some new oscillation criteria for second order neutral type difference equation of the form

$$
\Delta\left(a_{n}\left(\Delta z_{n}\right)^{\alpha}\right)+q_{n} f\left(x_{n}\right)=e_{n}, \quad n \geq n_{0}>0
$$

where $z_{n}=x_{n}-\rho_{n} x_{n-l}$ and $\alpha$ is ratio of odd positive integers. Examples are provided to illustrate the results.

2010 Mathematics Subject Classification: 39A10.

Key Words: Almost oscillation, second order, neutral difference equation.

\section{INTRODUCTION}

In this paper, we study the oscillatory behavior of second order neutral type difference equation of the form

$$
\Delta\left(a_{n}\left(\Delta z_{n}\right)^{\alpha}\right)+q_{n} f\left(x_{n}\right)=e_{n}, \quad n \geq n_{1}>0
$$

where $z_{n}=x_{n}-\rho_{n} x_{n-l}$ and $\alpha>0$ is a ratio of odd positive integers, $l$ is a positive integer.

Subject to the following hypothesis:

$\left(\mathrm{H}_{1}\right)\left\{\rho_{n}\right\},\left\{q_{n}\right\}$ and $\left\{e_{n}\right\}$ are sequences of real numbers with $0 \leq \rho_{n} \leq \rho<1, q_{n}>0, e_{n} \geq 0$ and $\left\{a_{n}\right\}$ is a positive real sequence with $\sum_{s=n_{0}}^{n} \frac{1}{a_{s}^{1 / \alpha}} \rightarrow \infty$ as $n \rightarrow \infty$.

$\left(\mathrm{H}_{2}\right) \quad f: R \rightarrow R$ such that $u f(u)>0$ for all $u \neq 0$ and there exists a positive constant $M$ such that $\frac{f(u)}{u^{\beta}} \geq M$ for all $u \neq 0$, where $\beta$ is a positive constant.

By a solution of equation (1), we mean a real sequence $\left\{x_{n}\right\}$ defined for all $n \geq n_{0}$ and satisfying equation (1). A solution $\left\{x_{n}\right\}$ of equation (1) is said to be oscillatory if it is neither eventually positive nor eventually negative, otherwise it is said to be non-oscillatory.

Recently there has been an increasing interest in the study of the oscillation and non-oscillation of the second order neutral difference equations, see for example [6, 7, 10-14] and the references cited there in. In [14], we see that the oscillation criteria for second order non-positive neutral term of the form

$$
\Delta\left(a_{n}\left(\Delta z_{n}\right)^{\alpha}\right)+q_{n} f\left(x_{n-\sigma}\right)=0, \quad n \geq n_{0}>0,
$$

where $z_{n}=x_{n}-\rho_{n} x_{n-l}$ with $\sum_{s=n_{0}}^{n} \frac{1}{a_{s}^{1 / \alpha}} \rightarrow \infty$ as $n \rightarrow \infty$ and $\frac{f(u)}{u^{\alpha}} \geq M$.

In [6] the authors studied oscillation criteria for second order neutral difference equation of the form 


$$
\Delta\left(r_{n}\left(\Delta x_{n}+x_{n-k}\right)^{\gamma}\right)+q_{n} x_{n+1}^{\alpha}=e_{n}
$$

In section 2, we present some oscillation criteria for equation (1) and in section 3, we provide some examples to illustrate the main results.

Definition $1.1 A$ solution $\left\{x_{n}\right\}$ of equation (1) is said to be almost oscillatory if either $\left\{x_{n}\right\}$ is oscillatory or $\Delta x_{n}$ is oscillatory or $x_{n} \rightarrow 0$ as $n \rightarrow \infty$.

We provide two lemmas which are useful in proving the main results.

Lemma 1.1 Set $F(x)=a x^{\alpha-\gamma}+\frac{b}{x^{\gamma}}$ for $x>0$. If $a>0, b>0$ and $\alpha>\gamma \geq 1$, then $F(x)$ attains its minimum

$$
F_{\min }=\frac{\alpha a^{\gamma / \alpha} b^{1-\gamma / \alpha}}{\gamma^{\gamma / \alpha}(\alpha-\gamma)^{1-\gamma / \alpha}}
$$

Lemma 1.2 For all $x \geq y \geq 0$ and $\gamma \geq 1$, we have the following inequality

$$
x^{\gamma}-y^{\gamma} \geq(x-y)^{\gamma}
$$

\section{ALMOST OSCILLATION RESULTS}

In this section, we establish new almost oscillation criteria for equation (1).

Theorem 2.1 Assume that there exists a sequence $\left\{P_{n}\right\}$ such that

$$
\limsup _{n \rightarrow \infty} \sum_{s=n_{0}}^{n-1} P_{s} Q_{s}^{*}-\frac{\left(\Delta P_{s}\right)^{\alpha+1}\left(P_{s+1}\right)^{1 / \alpha} a_{s}}{(\alpha+1)^{\alpha+1} P_{s}^{\alpha}}=\infty
$$

and

$$
\sum_{s=n_{0}}^{n-1} \sum_{u=n_{0}}^{s-1}\left(M b^{\beta} q_{u} \pm e_{u}\right)^{\frac{1}{\alpha}}=\infty
$$

where

$$
\begin{aligned}
Q_{n} & =\frac{\beta M^{\alpha / \beta} q_{n}^{\alpha / \beta} e^{1-\alpha / \beta}\left(1+\rho_{n}\right)^{\alpha}}{\alpha^{\alpha / \beta}(\beta-\alpha)^{1-\alpha / \beta}}, \\
Q_{n}^{*} & =\min \left\{Q_{n}, d^{\beta-\alpha}\left(1+\rho_{n}\right) M q_{n}-d^{-\alpha} e_{n}\right\},
\end{aligned}
$$

$M>0$ and $d>0$. Then every solution of equation (1) is almost oscillatory.

Proof. Suppose that sequence $\left\{x_{n}\right\}$ is not almost oscillatory solution of equation (1). There exists a positive solution $\left\{x_{n}\right\}$ of a equation (1) such that $x_{n-l}>0$ and $x_{n}>0$ for all $n \geq n_{1} \geq n_{0}$. Then by definition of almost oscillatory there are two possible cases arise.

Case I: Assume that $\Delta x_{n}>0$ for all $n \geq n_{1}$. Thus $\Delta z_{n}>0$ for all $n \geq n_{1}$. From the definition of $z_{n}$, we have $z_{n}=x_{n}-\rho_{n} x_{n-l}$ and $x_{n} \geq\left(1+\rho_{n}\right) z_{n}$. Then from equation (1) and $\left(H_{2}\right)$, we have

$$
\Delta\left(a_{n}\left(\Delta z_{n}\right)^{\alpha}\right)+M q_{n}\left(1+\rho_{n}\right)^{\beta} z_{n}^{\beta} \leq e_{n}
$$




$$
\Delta\left(a_{n}\left(\Delta z_{n}\right)^{\alpha}\right)+M q_{n}\left(1+\rho_{n}\right)^{\beta} z_{n}^{\beta} \leq e_{n}
$$

From the above inequality, we conclude that

$$
a_{n}\left(\Delta z_{n}\right)^{\alpha} \geq a_{n+1}\left(\Delta z_{n+1}\right)^{\alpha} .
$$

Let us denote the sequence $\left\{w_{n}\right\}$ by the following

$$
\begin{aligned}
w_{n} & =\frac{P_{n} a_{n}\left(\Delta z_{n}\right)^{\alpha}}{z_{n}^{\alpha}} \cdot n \geq n_{1} . \\
\Delta w_{n} & =\frac{P_{n} \Delta\left(a_{n}\left(\Delta z_{n}\right)^{\alpha}\right)}{z_{n}^{\alpha}}+a_{n+1}\left(\Delta z_{n+1}\right)^{\alpha} \Delta\left(\frac{P_{n}}{z_{n}^{\alpha}}\right), \\
& =\frac{P_{n} \Delta\left(a_{n}\left(\Delta z_{n}\right)^{\alpha}\right)}{z_{n}^{\alpha}}+a_{n+1}\left(\Delta z_{n+1}\right)^{\alpha}\left[\Delta\left(\frac{1}{z_{n}^{\alpha}}\right) P_{n}+\frac{1}{z_{n+1}^{\alpha}} \Delta P_{n}\right], \\
& =\frac{P_{n} \Delta\left(a_{n}\left(\Delta z_{n}\right)^{\alpha}\right)}{z_{n}^{\alpha}}+\frac{\Delta P_{n} a_{n+1}\left(\Delta z_{n+1}\right)^{\alpha}}{z_{n+1}^{\alpha}}-\frac{P_{n} a_{n+1}\left(\Delta z_{n+1}\right)^{\alpha} \Delta\left(z_{n}^{\alpha}\right)}{z_{n}^{\alpha} z_{n+1}^{\alpha}} .
\end{aligned}
$$

By mean value theorem, there exists $\xi \in\left(z_{n}, z_{n+1}\right)$ such that

$$
\Delta\left(z_{n}^{\alpha}\right)=\alpha \xi^{\alpha-1} \Delta z_{n}
$$

we have

$$
\Delta w_{n}=\frac{P_{n} \Delta\left(a_{n}\left(\Delta z_{n}\right)^{\alpha}\right)}{z_{n}^{\alpha}}+\Delta p_{n} \frac{w_{n+1}}{p_{n+1}}-\alpha p_{n} \frac{\xi^{\alpha-1} a_{n+1}\left(\Delta z_{n+1}\right)^{\alpha} \Delta z_{n}}{z_{n+1}^{\alpha} z_{n}^{\alpha}} .
$$

In the view of (9), (10) and (11), we obtain

$$
\Delta w_{n} \leq-P_{n}\left[M q_{n}\left(1+\rho_{n}\right)^{\beta} z_{n}^{\beta-\alpha}-\frac{e_{n}}{z_{n}^{\alpha}}\right]+\Delta P_{n} \frac{w_{n+1}}{p_{n+1}}-\frac{\alpha P_{n} \xi^{\alpha} a_{n+1}\left(\Delta z_{n+1}\right)^{\alpha} \Delta z_{n}}{\xi z_{n+1}^{\alpha} z_{n}^{\alpha}}
$$

and

$$
\Delta w_{n} \leq-P_{n}\left[M q_{n}\left(1+\rho_{n}\right)^{\beta} z_{n}^{\beta-\alpha}-\frac{e_{n}}{z_{n}^{\alpha}}\right]+\Delta P_{n} \frac{w_{n+1}}{P_{n+1}}-\frac{\alpha P_{n} w_{n+1}^{1+1 / \alpha}}{P_{n+1}^{1+1 / \alpha} a_{n}^{1 / \alpha}}
$$

Set

$$
F(u)=M q_{n}\left(1+\rho_{n}\right)^{\beta} u^{\beta-\alpha}-\frac{e_{n}}{u^{\alpha}}
$$

since $u$ is increased, there is a constant $d>0$ such that $u \geq d>0$ and

$$
F(u) \geq d^{\beta-\alpha}\left(1+\rho_{n}\right)^{\beta} M q_{n}-d^{-\alpha} e_{n} .
$$

By using the inequality

$$
B u-A u^{1+1 / \alpha} \leq \frac{\alpha^{\alpha}}{(\alpha+1)^{\alpha+1}} \frac{B^{\alpha+1}}{A^{\alpha}}, \quad A>0,
$$

we have 


$$
\frac{\Delta P_{n}}{P_{n+1}} w_{n+1}-\frac{\alpha}{p_{n+1}^{1+1 / \alpha} a_{n}^{1 / \alpha}} w_{n+1}^{1+1 / \alpha} \leq \frac{\left(\Delta P_{n}\right)^{\alpha+1} P_{n+1}^{1 / \alpha} a_{n}}{(\alpha+1)^{\alpha+1} P_{n}^{\alpha}} .
$$

From inequalities (12), (13) and (14), we have

$$
\Delta w_{n} \leq-\left[P_{n} Q_{n}^{*}-\frac{\left(\Delta p_{n}\right)^{\alpha+1} P_{n+1}^{1 / \alpha} a_{n}}{(\alpha+1)^{\alpha+1} P_{n}^{\alpha}}\right] .
$$

Summing the inequality (16) from $n_{1}$ to $n-1$, we obtain

$$
\sum_{s=n_{1}}^{n-1}\left[P_{s} Q_{s}^{*}-\frac{\left(\Delta P_{s}\right)^{\alpha+1} P_{s+1}^{1 / \alpha} a_{s}}{(\alpha+1)^{\alpha+1} P_{s}^{\alpha}}\right] \leq w_{n_{1}}-w_{n} \leq w_{n_{1}}, \text { for all } n
$$

which is contradiction to (6).

Next, we assume that $x_{n}<0$ for all $n \geq n_{1}$. We use the transformation $y_{n}=-x_{n}$, then we have $\left\{y_{n}\right\}$ is an eventually positive solution of equation

$$
\Delta\left(a_{n}\left(\Delta z_{n}\right)^{\alpha}\right)+q_{n} f\left(y_{n}\right)=-e_{n},
$$

where $z_{n}=y_{n}-\rho_{n} y_{n-l}>0$. Define

$$
w_{n}=\frac{P_{n} a_{n}\left(\Delta z_{n}\right)^{\alpha}}{z_{n}^{\alpha}}, n \geq n_{1} .
$$

Thus $w_{n}>0$ and satisfies

$$
\Delta w_{n} \leq-P_{n}\left[M q_{n}\left(1+\rho_{n}\right)^{\beta} z_{n}^{\beta-\alpha}+\frac{e_{n}}{z_{n}^{\alpha}}\right]+\Delta P_{n} \frac{w_{n+1}}{P_{n+1}}-\frac{\alpha P_{n} w_{n+1}^{1+1 / \alpha}}{P_{n+1}^{1+1 / \alpha} a_{n}^{1 / \alpha}} .
$$

Set

$$
F(u)=M q_{n}\left(1+\rho_{n}\right)^{\beta} u^{\beta-\alpha}+\frac{e_{n}}{u^{\alpha}} .
$$

Using Lemma 1.1, we see that

$$
F(u) \geq \frac{\beta q_{n}^{\alpha / \beta} M^{\alpha / \beta}\left(1+\rho_{n}\right)^{\alpha} e_{n}^{1-\alpha / \beta}}{\alpha^{\alpha / \beta}(\beta-\alpha)^{1-\alpha / \beta}}
$$

and also (14) holds. Then the rest of the proof is similar to that of the above and hence is omitted.

Case II: Assume that $\Delta x_{n}<0$ for all $n \geq n_{1}$, then $\Delta z_{n}<0$ for all $n \geq n_{1}$. From $x_{n}>0$ and $\Delta x_{n}<0$, we obtain

$$
\lim _{n \rightarrow \infty} x_{n}=b>0 .
$$

Hence there exists $n_{2} \geq n_{1}$ such that $x_{n}^{\beta} \geq b^{\beta}$ for $n \geq n_{2}$. Therefore we have

$$
\Delta\left(a_{n}\left(\Delta z_{n}\right)^{\alpha}\right) \leq-M q_{n} b^{\beta}+e_{n} .
$$

Summing the last inequality from $n_{2}$ to $n-1$, we obtain 


$$
a_{n}\left(\Delta z_{n}\right)^{\alpha}<a_{n}\left(\Delta z_{n}\right)^{\alpha}-a_{n_{2}}\left(\Delta z_{n_{2}}\right)^{\alpha} \leq-\left[\sum_{s=n_{2}}^{n-1} M b^{\beta} q_{s}-e_{s}\right]
$$

and

$$
\Delta z_{n} \leq-\left[\sum_{s=n_{2}}^{n-1} M b^{\beta} q_{s}-e_{s}\right]^{1 / \alpha} a_{n}^{-1 / \alpha}
$$

Again summing the above inequality from $n_{2}$ to $n-1$, we obtain

$$
z_{n} \leq z_{n_{2}}-\left[\sum_{s=n_{2}}^{n-1}\left[\sum_{u=n_{2}}^{s-1} M b^{\beta} q_{u}-e_{u}\right]^{1 / \alpha} a_{s}^{-1 / \alpha}\right]
$$

Letting $n \rightarrow \infty$, from condition (7) implies that $z_{n}$ is negative for all $n \geq n_{2}$, a contradiction.

Finally, we assume that $\left\{x_{n}\right\}$ is an eventually negative sequence. It means that there exists $n_{3} \in N$ such that $x_{n}<0$ for all $n \geq n_{3}$. We use the transformation $y_{n}=-x_{n}$ in equation (1). Then $y_{n}$ is an eventually positive solution of the equation

$$
\Delta\left(a_{n}\left(\Delta z_{n}\right)^{\alpha}\right)+q_{n} f\left(y_{n}\right)=-e_{n}
$$

where $z_{n}=y_{n}-\rho_{n} y_{n-l}$. The rest of the proof is similar to the above and hence omitted. The proof is now complete.

Corollary 2.1. Assume that all the conditions of Theorem 2.1 hold except the condition (6) is replaced by

$$
\limsup _{n \rightarrow \infty} \sum_{s=n_{0}}^{n} P_{s} Q_{s}^{*}=\infty
$$

and

$$
\limsup _{n \rightarrow \infty} \sum_{s=n_{0}}^{n} a_{s} \frac{\left(\Delta P_{s}\right)^{\alpha+1}}{P_{s}^{\alpha}} P_{s+1}^{1 / \alpha}<\infty
$$

Then every solution of equation (1) is almost oscillatory.

Theorem 2.2. Assume that condition (7) holds. Furthermore, assume that there exist a positive sequence $\left\{p_{n}\right\}$ and a double sequence $\left\{H_{m, n}: m \geq n \geq 0\right\}$ such that $H_{m, n}=0$ for $m \geq 0, H_{m, n}>0$ for $m>n>0$ and $\Delta_{2} H_{m, n}=H_{m, n+1}-H_{m, n} \leq 0$ for $m \geq n \geq 0$. If

$$
\frac{1}{H_{m, n}} \sum\left[H_{m, s} P_{s} Q_{s}^{*}-\frac{P_{s+1}^{1+\alpha} a_{s}\left(\Delta_{2} H_{m, s}+H_{m, s} \frac{\Delta P_{s}}{P_{s+1}}\right)^{\alpha+1}}{(\alpha+1)^{\alpha+1} H_{m, s}^{\alpha} P_{s}^{\alpha}}\right]=\infty
$$

Then every solution of equation (1) is almost oscillatory.

Proof. Proceeding as in Theorem 2.1, we have two cases to consider. 
Case I: Assume that $\Delta x_{n}>0$ for all $n \geq n_{1}$. Define $w_{n}$ by (11), then $w_{n}>0$ and satisfies

$$
\Delta w_{n} \leq-P_{n} Q_{n}^{*}+\frac{\Delta P_{n} w_{n+1}}{P_{n+1}}-\frac{\alpha P_{n} w_{n+1}^{1+1 / \alpha}}{P_{n+1}^{1+1 / \alpha} a_{n}^{1 / \alpha}} .
$$

Multiply both side by $H_{m, n}$ and summing from $n_{1}$ to $n-1$, we have

$$
\begin{aligned}
& \sum_{s=n_{1}}^{n-1} H_{m, s} \Delta w_{s} \leq-\sum_{s=n_{1}}^{n-1} H_{m, s} P_{s} Q_{s}^{*}+\sum_{s=n_{1}}^{n-1} H_{m, s} \Delta P_{s} \frac{w_{s+1}}{P_{s+1}}-\alpha \sum_{s=n_{1}}^{n-1} H_{m, s} \frac{P_{s} w_{s+1}^{1+1 / \alpha}}{P_{s+1}^{1+1 / \alpha} a_{s}^{1 / \alpha}} \\
& \sum_{s=n_{1}}^{n-1} H_{m, s} P_{s} Q_{s}^{*} \leq-\sum_{s=n_{1}}^{n-1} H_{m, s} \Delta w_{s}+\sum_{s=n_{1}}^{n-1} H_{m, s} \Delta P_{s} \frac{w_{s+1}}{P_{s+1}}-\alpha \sum_{s=n_{1}}^{n-1} H_{m, s} \frac{P_{s} w_{s+1}^{1+1 / \alpha}}{P_{s+1}^{1+1 / \alpha} a_{s}^{1 / \alpha}}
\end{aligned}
$$

Using summation by parts, we obtain

$$
\sum_{s=n_{1}}^{n-1} H_{m, s} P_{s} Q_{s}^{*} \leq H_{m, n} w_{n_{1}}+\sum_{s=n_{1}}^{n-1}\left[\left[\Delta_{2} H_{m, s}+H_{m, s} \frac{\Delta P_{s}}{P_{s+1}}\right] w_{s+1}-\alpha H_{m, s} \frac{P_{s} w_{s+1}^{1+1 / \alpha}}{P_{s+1}^{1+1 / \alpha} a_{s}^{1 / \alpha}}\right] .
$$

Setting $B=\Delta_{2} H_{m, s}+H_{m, s} \frac{\Delta P_{s}}{P_{s+1}}, \quad A=\frac{\alpha H_{m, s} P_{s}}{P_{s+1}^{1+1 / \alpha} a_{s}^{1 / \alpha}} \quad$ and $\quad u=w_{s+1}$

From inequalities (31) and (14), we obtain

$$
\begin{gathered}
\sum_{s=n_{1}}^{n-1}\left[H_{m, s} P_{s} Q_{s}^{*}-\frac{P_{s+1}^{1+\alpha}\left(\Delta_{2} H_{m, s}+H_{m, s} \frac{\Delta P_{s}}{P_{s+1}}\right)^{\alpha+1} a_{s}}{(\alpha+1)^{\alpha+1} H_{m, s}^{\alpha} P_{s}^{\alpha}}\right] \leq H_{m, n} w_{n_{1}}, \\
\frac{1}{H_{m, n}} \sum_{s=n}^{n-1}\left[H_{m, s} P_{s} Q_{s}^{*}-\frac{P_{s+1}^{1+\alpha}\left(\Delta_{2} H_{m, s}+H_{m, s} \frac{\Delta P_{s}}{P_{s+1}}\right)^{\alpha+1} a_{s}}{(\alpha+1)^{\alpha+1} H_{m, s}^{\alpha} P_{s}^{\alpha}}\right] \leq w_{n_{1}},
\end{gathered}
$$

which contradicts the assumption (28).

Next we consider the case when $x_{n}<0$ for all $n \geq n_{1}$. We use the transformation $y_{n}=-x_{n}$ then $y_{n}$ is a positive solution of equation

$$
\Delta\left(a_{n}\left(\Delta z_{n}\right)^{\alpha}\right)+q_{n} f\left(x_{n}\right)=-e_{n}
$$

when $z_{n}=y_{n}-\rho_{n} y_{n-l}$. Define $w_{n}$ by (18) and (20) hold. The remaing of the proof is similar to that of first case of Theorem 2.1 and hence omitted. The proof of the case II is similar to that of second case of Theorem 2.1. The proof is now complete.

Corollary 2.2. Assume that all the conditions of Theorem 2.2 hold except the condition (28) is replace by

$$
\limsup _{n \rightarrow \infty} \frac{1}{H_{m, n}} \sum_{s=n_{0}}^{n-1} H_{m, s} P_{s} Q_{s}^{*}=\infty
$$

and 


$$
\limsup _{n \rightarrow \infty} \frac{1}{H_{m, n}} \sum_{s=n}^{n-1} \frac{P_{s+1}^{1+\alpha}\left(\Delta_{2} H_{m, s}+H_{m, s} \frac{\Delta P_{s}}{P_{s+1}}\right)^{\alpha+1} a_{s}}{H_{m, s}^{\alpha} P_{s}^{\alpha}}<\infty .
$$

Then every solution of equation (1) is almost oscillatory.

\section{Examples}

In this section, we provide three examples.

Example 3.1. Let us consider the second order neutral difference equation of the form

$$
\Delta\left(2-\frac{(-1)^{n}}{n} \Delta\left(x_{n}-\frac{1}{2} x_{n-1}\right)\right)+x_{n}^{3}=\frac{3}{n(n+1)}-13(-1)^{n}, n \geq 2 .
$$

Here $a_{n}=2-\frac{(-1)^{n}}{n}, \quad \rho_{n}=\frac{1}{2}, \alpha=1, l=1, q_{n}=1, \quad f(u)=u^{3}$ and $e_{n}=\frac{3}{n(n+1)}-13(-1)^{n}$. All the conditions of Theorem 2.1 are satisfied. Hence every solution of the equation (34) is almost oscillatory. In fact one such solution is $(-1)^{n+1}$. Here $\left\{x_{n}\right\}$ is oscillatory.

Example 3.2. Let us consider the second order neutral difference equation of the form

$$
\Delta\left(2+(-1)^{n}\left(\Delta\left(x_{n}-\frac{1}{2} x_{n-1}\right)\right)\right)+x_{n}^{3}=14+25(-1)^{n}, \quad n \geq 2 .
$$

Here $a_{n}=2+(-1)^{n}, \rho_{n}=\frac{1}{2}, \alpha=1, l=1, q_{n}=1, f(u)=u^{3}$ and $e_{n}=14+25(-1)^{n}$. All the conditions of Theorem 2.1 are satisfied. Hence every solution of the equation (35) is almost oscillatory. In fact one such solution is $x_{n}=2-(-1)^{n+1}$. Here $\left\{x_{n}\right\}$ is nonoscillatory but $\Delta x_{n}$ is oscillatory.

Example 3.3. Let us consider the second order neutral difference equation of the form

$$
\Delta\left(\frac{1}{n+3} \Delta\left(x_{n}-2 x_{n-1}\right)\right)+n^{2} x_{n}^{3}=\frac{(n-1)(n+1)(n+2)-3}{(n-1) n(n+1)(n+2)}, n \geq 2 .
$$

Here $a_{n}=\frac{1}{n+3}, \rho_{n}=2, \alpha=1, l=1, q_{n}=n^{2}, f(u)=u^{3}$ and $e_{n}=\frac{(n-1)(n+1)(n+2)-3}{(n-1) n(n+1)(n+2)}$. All the conditions of Theorem 2.1 are satisfied. Hence every solution of the equation (36) is almost oscillatory. In fact one such solution is $x_{n}=\frac{1}{n}$. Here $\left\{x_{n}\right\}$ tends to zero as $n \rightarrow \infty$.

\section{References}

[1] R.P. Agarwal, Difference Equations and Inequalities, Second Edition, Marcel Dekker, New York, 2000.

[2] R.P. Agarwal, M. Bohner, S.R. Grace and D.'O. Regan, Discrete Oscillation Theory, Hindawi Publ. Corp., New York, 2005.

[3] R.P. Agarwal, M.M.S. Manuel and E. Thandapani, Oscillatory and nonoscillatory behavior of second order delay difference equations, Appl. Math. Lett., 10(2)(1997), 103-109. 
[4] J. Cheng, Existence of nonoscillatory solution of second order linear difference equations, Appl. Math. Lett., 20(2007), 892-899.

[5] I. Gyori and G. Ladas, Oscillation Theory of Delay Differential Equations with Applications, Clarendon Press, Oxford, 1991.

[6] R. Jankowski and E. Schmeidel, Almost oscillation criterca for second order neutral difference equations with quasidifferences, International Journal of Difference Equations, 9(1)(2014), 77-86.

[7] J. Jiang, Oscillation criteria for second order quasilinear neutral delay difference equations, Appl. Math. Comput., 125(2002), 287-293.

[8] W.G. Kelley and A.C. Peterson, Difference Equations: An Introduction with Applications, Acad. Press, New York, 1991.

[9] G. Ladas, Ch.G. Philos and Y.G. Sticas, Sharp condition for the oscillation of delay difference

equations, J. Appl. Math. Simul., 2(2)(1989), 101-112.

[10] H.J. Li and C.C. Yeh, Oscillation criteria for second order neutral delay difference equations, Comput. Math. Appl., 36(1999), 123-132.

[11] E. Thandapani, J.R. Graef and P.W. Spikes, On the oscillation of second order quasilinear difference Nonlinear World, 3(1996), 545-565.

[12] E. Thandapani and K. Mahalingam, Oscillation and nonoscillation of second order neutral difference equations, Czechoslovak Math. J., 53(128)(2003), 935-947.

[13] E. Thandapani and S. Selvarangam, Oscillation theorems for second order nonlinear neutral difference equations, J. Math. Comput. Sci., 2(4)(2012), 866-879.

[14] E. Thandapani, S. Selvarangam, R. Rama and M. Madhan, Improved oscillation criteria for second order nonlinear delay difference equations with nonpositive neutral term, Fasciculi Mathematici, 2016. 